\title{
Group structure of block transformations: Matching conditions for the critical properties of lattice spin systems
}

\author{
A. L. Stella, ${ }^{*}$ R. Dekeyser, and J. Indekeu \\ Instituut Voor Theoretische Fysica, Katholieke Universiteit Leuven, \\ Celestijnenlaan 200 D, B-3030 Leuven, Belgium \\ F. Toigo \\ Istituto di Fisica e Unità, Gruppo Nazionale di Struttura della Materia del Consiglio Nazionale delle Ricerche, \\ Università di Padova, Padova, Italy
}

(Received 29 May 1979)

\begin{abstract}
Matching procedures, related to the group structure of block-spin renormalization, are proposed for an optimal choice of parameter-dependent transformations. The method also allows to determine the parameter's dependence on the reduced coupling near the fixed point. Applications to linear transformations for two- and three-dimensional Ising models and twodimensional three-, four-, and five-state Potts systems are reported. With simple approximations good estimates of critical couplings and exponents are obtained. Results for the twodimensional spin- $\frac{1}{2} X Y$ system and their possible implications, as far as an eventual transition is concerned, are discussed. Some applications to nonlinear transformations are also considered.
\end{abstract}

\section{INTRODUCTION}

A general and important problem in the positionspace renormalization approach ${ }^{1,2}$ is that of choosing an appropriate transformation. For example, how should one fix the values of free (or apparently free) parameters entering the definition of the transformation? The present paper is primarily concerned with this problem.

A typical case is that of linear transformations, ${ }^{3}$ which should possess nontrivial short-range fixed points only for a well-defined value of the specifying parameter; in approximations, however, we generally get fixed points, with different critical behaviors, for a whole range of values of this parameter. Apart from this example, even in the case where we a priori expect a certain amount of freedom in the definition of the transformation, in practice we experience that the approximate critical properties predicted by the approach never show independence from the parameters determining the transformation, as they should.

A possible strategy in this kind of situation is to exploit the freedom in the specification of the transformation, in such a way as to optimize it, within a given approximation; this must be done following an appropriate criterion, capable of giving us finally unique and, possibly, reliable results as far as critical properties are concerned.

In cases like that of linear transformations, a criterion for fixing the optimal transformation has the additional important significance of yielding the best approximate realization of its unique allowed determination.

With this work we propose a new kind of optimali- zation criterion, based on very general group structure properties of block-spin renormalization; we test this criterion on a large variety of spin systems and transformations with rather satisfactory results, if compared with those given by different methods of similar complexity. In its simplest version this criterion was already discussed in a previous note. ${ }^{4}$

The most widely applied and successful optimalization procedure, in the last few years, has certainly been the one introduced by Kadanoff et al. ${ }^{5}$ : In their method an approximate transformation is constructed, generating a free energy with a rigorous upper or lower bound character with respect to the true one; the parameters are then fixed such as to optimize this bound. In spite of some impressive successes in the determination of critical exponents, after a more careful analysis these variational procedures turned out to be seriously limited as far as a fully consistent applicability is concerned. ${ }^{6}$ This is due to the fact that in certain cases singularities can start developing in the transformation, when the parameters are made coupling dependent. Only quite recently a new version of this variational method has been devised, ${ }^{7}$ working within the limits of the first-order cumulant expansion, but not suffering of the above-mentioned pathology.

The merit of the approach presented in the present paper, in our opinion, is not limited to the immediate practical advantages, like the very accurate predictions for the critical temperature. It is certainly an equally important achievement that it allows us to write the optimal parameter as a function of the coupling strength near criticality, without contradicting the basic assumptions of regularity of the renormali- 
zation approach. Indeed, this determination turned out to be possible in all cases, giving also rise to quite reasonable results for the exponents.

Unlike the above-mentioned variational approach, the equations determining the behavior of the parameter as a function of the coupling do not contain derivatives of the free energy, which is singular at criticality; they only contain derivatives of renormalization transformations, because they express constraints originating from the group structure of the transformations themselves. This indeed makes our method safe from the danger of singularities confluent in the transformation.

The plan of the paper is as follows. In Secs. II and III we present the main features of our matching procedures and outline the typical context of their application. In Sec. IV a comment is given on the results contained in Tables I, II, III, and IV; these results refer to two- and three-dimensional Ising and two-dimensional three-, four-, and five-state Potts. systems, and are obtained working mostly with strictly linear transformations. Some applications to nonlinear transformations are discussed in Sec. V; for these results a comparison can be made with what one finds following the free-energy optimalization strategy. In Sec. VI we report about an instructive application of the method to the puzzling spin- $\frac{1}{2} X Y$ system in two dimensions. The paper concludes with Sec. VII, in which some difficulties are also discussed and further possible developments are outlined.

\section{MODELS AND RENORMALIZATION TRANSFORMATIONS}

Except for the case of the two-dimensional spin- $\frac{1}{2}$ $X Y$ model, to be discussed in Sec. VI, we are concerned with classical, $s$-state Potts-like systems on two- and three-dimensional lattices. At each lattice site, $i$, a variable $\sigma_{i}$ can take on $s$ different values, say $\sigma_{i}=1,2, \ldots, s$, and the reduced nearestneighbor Hamiltonian $H$, has the form

$$
H=-\beta \mathcal{H}=\frac{s}{s-1} K \sum_{\langle j\rangle}\left(\delta_{\sigma_{i}, \sigma_{j}}-\frac{1}{s}\right),
$$

where $\beta^{-1}=k_{B} T, \delta$ is a Kronecker delta, and $\langle i j\rangle$ means restriction over nearest-neighbor pairs. In the following, for the calculation of magnetic exponents, we will eventually consider small external field perturbations added to Eq. (1), of the form

$$
\frac{s}{s-1} h \sum_{i}\left(\delta_{\sigma_{i}, 1}-\frac{1}{s}\right) \text {. }
$$

The above systems can also be viewed as a kind of $(s-1)$-dimensional vector spin model, according to the relation

$$
\delta_{\sigma_{i}, \sigma_{j}}=s^{-1}\left[1+(s-1)\left(\vec{\sigma}_{i}, \vec{\sigma}_{j}\right)\right],
$$

where $($,$) indicates scalar product and \vec{\sigma}_{i}, \vec{\sigma}_{j}$ are unit vectors which can only point to the vertices of an $(s-1)$-dimensional hypertetrahedron in spin space. In this language Eq. (2.1) becomes

$$
H=H(\{\vec{\sigma}\})=K \sum_{\langle i j\rangle}\left(\vec{\sigma}_{i}, \vec{\sigma}_{j}\right) .
$$

For $s=2$, Eqs. (2.1) and (2.1') reduce to the most familiar Ising Hamiltonian.

The first step, in the standard setup of a real-space renormalization scheme, ${ }^{1}$ is that of dividing the original lattice, with lattice distance $a$, into blocks of $l^{d}$ spins ( $d$ is spatial dimensionality), forming together a lattice of the original type, but with rescaled distance $a^{\prime}=a l$. New variables, $\vec{\mu}_{\alpha}$, are then associated with these cells, numbered by $\alpha$. A weight function $P(\{\vec{\mu}\},\{\vec{\sigma}\})$ is defined, satisfying certain positivity and normalization constraints; the renormalized Hamiltonian $H^{\prime}(\{\vec{\mu}\})$ is then obtained through the basic relation,

$$
H^{\prime}(\{\vec{\mu}\})=\ln \left[\operatorname{Tr}_{\{\vec{\sigma} \mid} e^{H(|\vec{\sigma}|)} P(\{\vec{\mu}\},\{\vec{\sigma}\})\right],
$$

the trace being over all $\vec{\sigma}$ spin configurations.

The nature of an operation like Eq. (2.3) is such that it in general maps an initial nearest-neighbor Hamiltonian into a much more complicated one, with an infinite number of interactions, in principle ${ }^{8}$; thus Eq. (2.3) actually induces a transformation in an infinite dimensional coupling parameter space. In approximations, however, only truncated versions of the full transformation are realized, and in the following we will restrict ourselves to the most simple ones, namely those involving only the nearestneighbor interaction $K$. Complications met in applying the method to transformations acting in higher dimensional spaces are discussed in Sec. VII.

We will mainly deal with one-parameter-dependent weight functions of the linear type, which for Pottslike systems can be written, indicating by $q$ this parameter,

$$
P_{q}(\{\vec{\mu}\},\{\vec{\sigma}\})=\prod_{\alpha} \frac{1}{2}\left(1+q \sum_{i \in \alpha}\left(\vec{\mu}_{\alpha}, \vec{\sigma}_{i}\right)\right\} .
$$

A different, nonlinear type of weight function will be considered in Sec. V.

The (approximate) transformation induced by weights like Eq. (2.4) in the interaction space, according to Eq. (2.3), will be written

$$
K^{\prime}=f^{(l)}(K, q) \text {, }
$$

where for clarity the rescaling factor associated with the transformation is explicitly reported as superscript. We practically find that the approximate transformation (2.5) allows in general for an infinite set of nontrivial fixed point solutions,

$$
K^{*}=f^{(l)}\left(K^{*}, q\right)
$$


corresponding to different values of the parameter $q$, $q=q^{(l)}\left(K^{*}\right)$. Moreover these fixed points are not at all physically equivalent, giving rise to different critical temperatures and exponents.

These circumstances lead to the need of a criterion for fixing the parameter $q$; this goes together with the hope that this criterion also chooses the "best" set of results among the whole infinity of possibilities which are left by Eq. (2.5).

\section{GROUP STRUCTURE AND MATCHING PROCEDURES}

In this section we want to show how the group structure properties, implicit in every block-spin renormalization approach, can naturally provide this criterion.

Resummation schemes like that of Eq. (2.3), typical of the construction of renormalization transformations, always imply the existence of semigroup structures for the transformations themselves. For transformations in real space, the semigroup is generally a discrete one, with elements given by all the powers of an initial generating transformation. Using here the notation $P_{q}$ for indicating a general, not necessarily linear, $q$-dependent weight function, its $n$th power $\left(P_{q}\right)^{n}(n=1,2, \ldots)$ is obtained from $P_{q}$ using Eq. (2.3); for example, with $n=2$ we have

$$
\left(P_{q}\right)^{2}(\{\vec{\nu}\},\{\vec{\sigma}\})=\operatorname{Tr}_{\{\vec{\mu} \mid} P_{q}(\{\vec{\nu}\},\{\vec{\mu}\}) P_{q}(\{\vec{\mu}\},\{\vec{\sigma}\}),
$$

where $\operatorname{Tr}_{\{\vec{\mu}\}}$ is a trace over the cell variables of Eq. (2.4), playing here the role of intermediate variables. By definition, indeed, $\left(P_{q}\right)^{2}$ associates $\vec{v}$ variables to cells of cells (described by $\vec{\mu}$ 's) of the initial $\vec{\sigma}$ spins, according to the same prescription as given by $P_{q}$.

The resulting rescaling factor associated with $\left(P_{q}\right)^{2}$ is thus $l^{2}$.

The linear weight has the useful property that the weight $\left(P_{q}\right)^{n}$ remains of linear type for every $n$; we have, e.g., that the square of Eq. (2.4) is simply

$$
\begin{aligned}
& \left(P_{q}\right)^{2}(\{\vec{v}\},\{\vec{\sigma}\})=\prod_{\Gamma} \frac{1}{2}\left(1+r(q) \sum_{i \in \Gamma}\left(\vec{\nu}_{\Gamma}, \vec{\sigma}_{i}\right)\right), \\
& r(q)=q^{2},
\end{aligned}
$$

$\Gamma$ labeling here cells of $\vec{\mu}$ cells, with $l^{2 d} \vec{\sigma}$ spins. If, particularizing the notations to this linear case, we indicate by

$$
K^{\prime}=f^{\left(l^{2}\right)}(K, r(q))
$$

the transformation associated with Eq. (3.2a), an obvious consequence of Eqs. (3.1) and (2.3) is that, if computed "exactly, ${ }^{18} f^{(l)}$ and $f^{\left(l^{2}\right)}$ should satisfy identically in $K$ and $q$ the following functional relationship':

$$
f^{\left(l^{2}\right)}(K, r(q)) \equiv f^{(l)}\left(f^{(l)}(K, q), q\right),
$$

which is nothing else but relation (3.1) between $\left(P_{q}\right)^{2}$ and $P_{q}$, as reflected in the corresponding transformations. A straightforward consequence of Eq. (3.4) is that, for every value of $q$, the fixed points of $f^{(l)}$ should also be fixed points of $f^{\left(l^{2}\right)}$.

As one can easily verify, however, if the two transformations are computed independently, using different approximations, the condition (3.4) turns out in general to be far from being satisfied. For example, if we indicate by $q^{\left(l^{2}\right)}\left(K^{*}\right)$ the fixed point value of $q$, as a function of $K^{*}$ for $f^{\left(l^{2}\right)}$, we find that this function and $q^{(l)}\left(K^{*}\right)$ are not coincident, and have even different ranges of definition. A typical situation, for $q^{(l)}$ and $q^{\left(l^{2}\right)}$, is illustrated in Fig. 1 .

It is thus an effect of approximations to violate group structure properties like Eq. (3.4). This circumstance suggests us in a natural way, as criterion for optimalization of the transformations, to choose for the parameter $q$ that value which fulfills the group structure properties in the closest possible way.

Such a choice in itself appears very plausible, if we realize that the group structure properties are directly connected with the iterative character of renormalization, which is of fundamental importance in the whole approach. We further note that, as mentioned in the Introduction, an optimalization of this kind, not involving other quantities than renormalization transformations, is particularly appealing, since the problem of confluent singularities is certainly avoided.

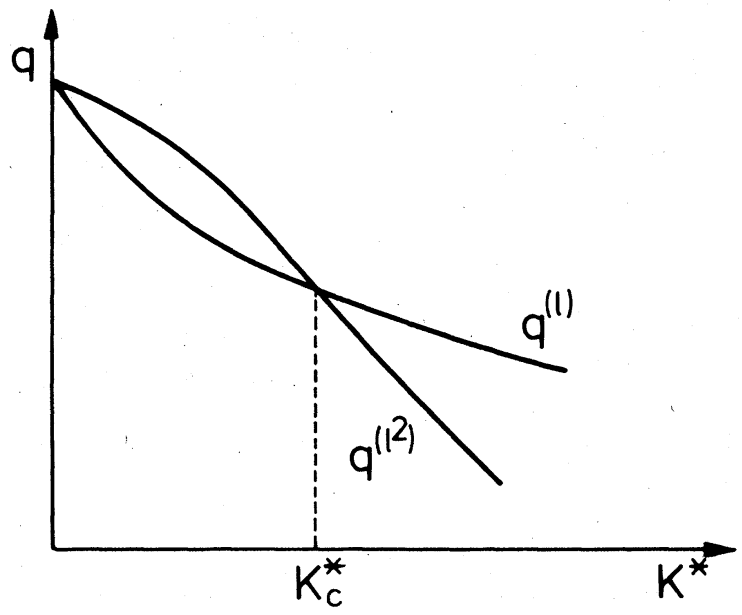

FIG. 1. Typical qualitative behavior of the functions $q^{(l)}$ and $q^{\left(l^{2}\right)}$ in the linear case; $K_{c}^{*}$ is the intersection abscissa, taken as best fixed point of the two transformations, $f^{(l)}$ and $f^{\left(l^{2}\right)}$. 
Our criterion, of course, has to be tested in many examples, in order to see whether or not it gives rise to reasonable results; this is indeed one of the main tasks to which the computational work reported here has been devoted.

According to what we said above, we will select, as "best" approximate fixed point for both $f^{(l)}$ and $f^{\left(l^{2}\right)}$, the one that corresponds to the intersection, if any, ${ }^{10}$ of the two curves $q^{(l)}\left(K^{*}\right)$ and $q^{\left(l^{2}\right)}\left(K^{*}\right)$ (see Fig. 1). In the following we will indicate by $K_{c}^{*}$ this abscissa of intersection.

We want to preserve the strictly linear character of the transformations, in particular the $K$ independence of the parameters $q$ and $r$; the matching just described is all we can do, being sufficient to determine completely and uniquely both transformations within these limits.

Apart from the magnetic ones, also the thermal eigenvalues of $f^{(l)}$ and $f^{\left(l^{2}\right)}$, at the common fixed point,

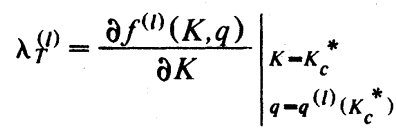

and

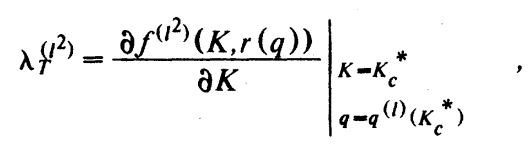

will not be simply related by a square power operation, as Eq. (3.4) would imply, but they will give rise to two different estimates of the thermal exponent $y_{T}$,

$$
y_{T}^{(l)}=\ln \lambda_{T}^{(l)} / \ln l
$$

and

$$
y_{T}^{\left(l^{2}\right)}=\ln \lambda T^{\left(l^{2}\right)} / \ln l^{2} .
$$

One may ask whether it is possible to modify and push further the above simple scheme, by requiring from the approximate transformations a more strict and extensive satisfaction of group structure constraints like Eq. (3.4). The answer, to which the rest of this section is devoted, is positive, as far as we accept to allow for a $K$ dependence of the parameters appearing in the transformations.

In the linear case, this amounts to a modification of the character of the transformations, which, strictly speaking, should afterwards be regarded as belonging to the more general class of nonlinear ones. ${ }^{1}$. This however should not cause trouble, because, at least in our opinion, there is nothing very fundamental in the distinction between linear and nonlinear transformations.
Restricting the discussion to the case of originally linear weights, let us thus assume that $q$ and $r$, the parameters of $P_{q}$ and $\left(P_{q}\right)^{2}$ in Eqs. (2.4) and (3.2), respectively, become functions of $K$. It is easily realized that the above-raised question then essentially amounts to ask whether it is possible to determine suitable $q(K)$ and $r(K)$ functions, such that the identity

$f^{\left(l^{2}\right)}(K, r(K))=f^{(l)}\left(f^{(l)}(K, q(K)), q\left(f^{(l)}(K, q(K))\right)\right)$

is satisfied, with our approximate $f^{(l)}$ and $f^{\left(1^{2}\right)}$, not only at the fixed point itself, but at least in an infinitesimal neighborhood of the fixed point.

Note that Eq. (3.7) on its own allows in principle a large variety of possible solutions for $q(K)$ and $r(K)$. It must be stressed, however, that the relation (3.1) between $P_{q}$ and $\left(P_{q}\right)^{2}$ imposes in the exact context a strong further limitation on the choice of the functions $q(K)$ and $r(K)$. Indeed, this further exact constraint, again originating from the group structure properties, gives us an additional equation for the determination of our unknown functions. In the case considered here (linear weights), relation (3.1) can be translated into the equation

$$
r(K) \equiv q(K) q\left(f^{(l)}(K, q(K))\right)
$$

Obtaining fully consistent solutions of the system Eqs. (3.7)-(3.8) for general $K$ is an extremely hard task. We will limit ourselves to study the behavior of $q$ and $r$ in the immediate neighborhood of the fixed point $K_{c}^{*}$. Note for this purpose that, if we impose

$$
r\left(K_{c}^{*}\right)^{1 / 2}=q\left(K_{c}^{*}\right)=q^{(l)}\left(K_{c}^{*}\right),
$$

where $q^{(l)}$ and $K_{c}^{*}$ have been previously defined, the Eqs. (3.7) and (3.8) above turn out to be automatically satisfied for $K=K_{c}^{*}$, which of course remains the common fixed point also of these modified transformations. If we now want to satisfy Eqs. (3.7) - (3.8) also in a small neighborhood of $K_{c}^{*}$, we must allow $r(K)$ and $q(K)$ to deviate slightly from their fixed point values:

$$
\begin{aligned}
& r(K)=r\left(K_{c}^{*}\right)+\left.\left(K-K_{c}^{*}\right) \frac{d r}{d K}\right|_{K_{c}^{*}}, \\
& q(K)=q\left(K_{c}^{*}\right)+\left.\left(K-K_{c}^{*}\right) \frac{d q}{d K}\right|_{K_{c}^{*}},
\end{aligned}
$$

and we have to determine both derivatives. We therefore differentiate Eqs. (3.7) and (3.8) at 
$K=K_{c}^{*}$ with respect to $K$, and we obtain

$$
\begin{aligned}
\left(\lambda_{T}^{\left(l, l^{2}\right)}\right)^{2}= & \left.\frac{\partial f^{\left(l^{2}\right)}}{\partial K}\right|_{K_{c}^{*}}+\left.\left.\frac{\partial f^{\left(l^{2}\right)}}{\partial r}\right|_{K_{c}^{*}} \frac{d r}{d K}\right|_{K_{c}^{*}} \\
= & \left(\left.\frac{\partial f^{(l)}}{\partial K}\right|_{K_{c}^{*}}+\left.\left.\frac{\partial f^{(l)}}{\partial q}\right|_{K_{c}^{*}} \frac{d q}{d K}\right|_{K_{c}^{*}}\right)^{2}, \quad\left(3.7^{\prime}\right) \\
\left.\frac{d r(K)}{d K}\right|_{K_{c}^{*}}= & \left.\left.\left.\left.q\left(K_{c}^{*}\right) \frac{d q}{d K}\right|_{K_{c}^{*}}\right|^{*}\right|_{K_{c}^{*}}+\left.\left.\frac{\partial f^{(l)}}{\partial q}\right|_{K_{c}^{*}} \frac{d q}{d K}\right|_{K_{c}^{*}}\right)
\end{aligned}
$$

The first equality in Eq. (3.7') is a definition of the new common thermal eigenvalue, $\lambda_{T}^{\left(l, l^{\prime}\right)}$. Equations $\left(3.7^{\prime}\right)$ and $\left(3.8^{\prime}\right)$ will in general allow us to determine the two unknown quantities $\left.(d r / d K)\right|_{K_{c}}{ }^{*}$ and $\left.(d q / d K)\right|_{K_{c}^{*}}$, and thus $\lambda_{T}^{\left(1, l^{2}\right)}$ and the corresponding exponent

$$
y_{t}^{\left(l, l^{2}\right)}=\ln \lambda_{t}^{\left(l, l^{2}\right)} / \ln l
$$

The nonlinear system of Eqs. (3.7') and (3.8') allows up to two real solutions, in general. At least in the cases considered, however, one of them could easily be excluded due to the corresponding abnormal magnitude of the derivatives. The degree of the system effectively reduces when $f^{(l)}$ and $f^{\left(l^{2}\right)}$ are computed in first-order cumulant approximation, in which case it has strictly only one solution.

As we will stress in Sec. V, the scheme outlined above is in principle not limited to the case of weights of the form (2.4). It should however be remarked that this particular form sensibly simplifies the calculations; it also allows some practical simplifications, which give the possibility of working with a relatively low number of degrees of freedom in some cases, as we will discuss in Sec. IV.

\section{RESULTS FOR POTTS-LIKE SYSTEMS IN TWO AND THREE DIMENSIONS}

Most of our test calculations have been devoted to various approximate linear transformations for Ising systems on the two-dimensional triangular and square, and the three-dimensional simple cubic lattices. The approximations used were the simple first-order cumulant expansion and the two-cell cluster calculation, ${ }^{1}$ which both work in the onedimensional nearest-neighbor coupling space. The smallest cells, with $l^{d}$ spins, were the triangular three-spin cell, the square four-spin cell, and the cubic eight-spin cell, respectively, for the abovementioned lattices. In the cumulant case, $f^{(l)}$ and $f^{\left(l^{2}\right)}$ were computed by taking as unperturbed Hamiltonians the intracell Hamiltonians of the $l^{d}$ and $l^{2 d}$ spin cells, respectively.

It should be noted, however, that in order to make the application possible to three dimensions, we had to conceive a variant of the scheme, which we discuss briefly below. Indeed, a cell of $2^{6}$ Ising spins is beyond any hope to be treated exactly with a computer. We managed to reduce this awkward number to 27 , corresponding to a decisively smaller $3 \times 3 \times 3$ cell. To explain this variant method in its simplest way, suppose we work on the square lattice with a weight of the form (2.4), and assume that $P_{q}$ groups the spins in square $2 \times 2$ cells. Instead of considering its second power $\left(P_{q}\right)^{2}$, which involves rather large $4 \times 4$ cells, let us look at a different linear weight $P_{r}^{\prime}$, with a parameter denoted by $r$ and smaller $3 \times 3$ cells.

It is clear that, even if both $P_{q}$ and $P_{r}^{\prime}$ are of the same linear form, and involve square cells, there is no simple algebraic connection between the two, like between $P_{q}$ and $\left(P_{q}\right)^{2}$. Their transformations cannot belong to the same semigroup, because 3 is not an integer multiple of 2 . However, if we consider $\left(P_{q}\right)^{n}$ and $\left(P_{r}^{\prime}\right)^{m}$, and allow both $m$ and $n$ to become very large, with the ratio $n / m$ approaching asymptotically the irrational limit $\ln 3 / \ln 2$, elementary arguments show us that the two power transformations should become as close to one another as we want, in this limit; the analog of Eq. (3.2b) is then

$$
r=(q)^{\ln 3 / \ln 2} \text {. }
$$

This suggests us to look for a matching of the fixed point value of $q, q^{(2)}\left(K^{*}\right)$, with the fixed point value of $r$, say $r^{(3)}\left(K^{*}\right)$, elevated to the power $\ln 2 / \ln 3$.

Along the same lines, it can be seen that in this case also the generalization to $K$-dependent parameters is possible; after some algebra one finds the following equations in $\left.(d q / d K)\right|_{K_{c}}{ }^{*}$ and $\left.(d r / d K)\right|_{K_{c}^{*}}$ :

$$
\begin{aligned}
& \left(\left.\frac{\partial f^{\left(l^{\prime}\right)}}{\partial K}\right|_{K_{c}^{*}}+\left.\left.\frac{\partial f^{\left(l^{\prime}\right)}}{\partial r}\right|_{K_{c}^{*}} \frac{d r}{d K}\right|_{K_{c}^{*}}\right)^{\ln l} \\
& =\left|\frac{\partial f^{(l)}}{\partial K}\right|_{K_{c}^{*}}+\left.\left.\left.\frac{\partial f^{(l)}}{\partial q}\right|_{K_{c}^{*}} \frac{d q}{d K}\right|_{K_{c}^{*}}\right|^{\ln l^{\prime}} \\
& =\left(\lambda f^{\left(l, l^{\prime}\right)}\right)^{\ln l^{\prime}}, \\
& \left.\frac{d r}{d K}\right|_{K_{c}^{*}}=\left.\frac{d q}{d K}\right|_{K_{c}^{*}} \frac{r\left(K_{c}^{*}\right)}{q\left(K_{c}^{*}\right)} \frac{1-\left(\lambda_{T}^{(l)}\right)^{\ln l^{\prime} / \ln l}}{1-\lambda_{T}^{(l)}},
\end{aligned}
$$

where $\lambda_{T}^{(l)}$ has been defined in Eq. (3.5), and we indicated by $l$ and $l$ the rescaling factors of the two transformations which are considered.

It is worth noting that the above-mentioned 
TABLE I. Results for the Ising model on the triangular lattice. The magnetic exponents $y_{h}^{(\sqrt{3})}$ and $y_{h}^{(3)}$ are obtained considering magnetic perturbations in the respective transformations at the fixed point. The exponent $y_{T}^{(\sqrt{3}, 3)}$ is obtained by allowing a $K$-dependent parameter $q$, as described by Eq. (3.11).

\begin{tabular}{|c|c|c|c|c|c|c|c|}
\hline & $q^{(\sqrt{3})}\left(K_{c}^{*}\right)$ & $K_{c}^{*}$ & $y_{T}(\sqrt{3})$ & $y_{h}^{(\sqrt{3})}$ & $y_{T}^{(3)}$ & $y_{h}^{(3)}$ & $y_{T}^{(\sqrt{3}, 3)}$ \\
\hline Cumulant & 0.437 & 0.257 & 1.114 & 1.578 & 1.057 & 1.540 & 0.875 \\
\hline Cluster & 0.445 & 0.250 & 1.080 & 1.618 & 1.032 & 1.632 & 0.857 \\
\hline Exact & & 0.275 & 1.000 & 1.875 & 1.000 & 1.875 & 1.000 \\
\hline
\end{tabular}

scheme is made possible not only by the symmetry of the lattice and of the cells; its applicability essentially relies on the peculiar character of the linear weights, which remain invariant in form under power raising.

In Tables I, II and III, results obtained by our various matching schemes for Ising models are reported and compared with data from exact solutions or approximate series methods.

We note that, even if sometimes the big cell calculations involve a rather large number of spins, this is compensated in some sense by the extreme simplicity of the approximations, leading to rather straightforward computer calculations. What is most satisfactory, in general, compared with the usual standard of real-space renormalization results, is the accuracy of the predicted critical couplings; as in the case of the $s=3,4$, and 5 Potts systems, discussed below, these always turn out to deviate only by a few percent from their exact or expected value. The estimates of the thermal exponents are also quite satis- factory in most of the cases, whereas the magnetic ones turn out to be poorer in general. The slight but definite tendency to improvement of $K_{c}^{*}$ and $\lambda \tau^{\left(1, l^{\prime}\right)}$, when one considers matchings between larger cells in the square lattice, is also worth noting (see Table II).

We used the same kind of matching procedures for the three-state Potts system on the triangular lattice, using three- and nine-spin cells like in the Ising case, and for the $s$-state system $(s=3,4$, and 5) on the square lattice with four- and nine-spin square cells; also in this case a cell of 16-spins with $s=5$ would not be treatable with the computer, so we turned to the above discussed variant.

We limited ourselves to give estimates of the thermal critical properties. As can be seen from Table IV, the agreement of the critical couplings with the exact ones ${ }^{11}$ is even more satisfactory than in the Ising, $s=2$, case. Also the trend of the $y_{T}$ exponents, with varying $s$, is in qualitative accordance with what should be expected. ${ }^{12}$

TABLE II. Results for the Ising model on the square lattice; both in the cluster and in the cumulant approximation case, each row corresponds to one of the three possible independent matchings that one can impose on the transformations $f^{(2)}, f^{(3)}$, and $f^{(4)}$.

\begin{tabular}{|c|c|c|c|c|c|c|c|c|c|}
\hline & 1 & $l^{\prime}$ & $q^{(l)}\left(K_{c}^{*}\right)$ & $K_{c}^{*}$ & $y_{T}^{(l)}$ & $y_{h}^{(l)}$ & $y_{T}^{\left(l^{\prime}\right)}$ & $y_{h}^{\left(l^{\prime}\right)}$ & $y_{T}^{\left(1, l^{\prime}\right)}$ \\
\hline \multirow{3}{*}{ Cumulant } & 2 & 3 & 0.327 & 0.409 & 1.209 & 1.682 & 1.164 & 1.655 & 0.955 \\
\hline & 2 & 4 & 0.325 & 0.413 & 1.212 & 1.698 & 1.142 & 1.655 & 0.956 \\
\hline & 3 & 4 & 0.165 & 0.417 & 1.172 & 1.696 & 1.148 & 1.679 & 0.964 \\
\hline \multirow{3}{*}{ Cluster } & 2 & 3 & 0.330 & 0.411 & 1.229 & 1.705 & 1.180 & 1.699 & 0.954 \\
\hline & 2 & 4 & 0.329 & 0.414 & 1.231 & 1.713 & 1.156 & 1.702 & 0.960 \\
\hline & 3 & 4 & 0.169 & 0.417 & 1.184 & 1.720 & 1.159 & 1.716 & 0.973 \\
\hline Exact & & & & 0.441 & 1.000 & 1.875 & 1.000 & 1.875 & 1.000 \\
\hline
\end{tabular}


TABLE III. Results for the three-dimensional Ising model. Contrary to the general trend, in this case the bigger-cell thermal exponents turn out to be slightly poorer than the small-cell ones.

\begin{tabular}{lccccccc}
\hline \hline & $q^{(2)}\left(K_{c}^{*}\right)$ & $K_{c}^{*}$ & $y_{T}^{(2)}$ & $y_{h}^{(2)}$ & $y_{T}^{(3)}$ & $y_{h}^{(3)}$ & $y_{T}^{(2,3)}$ \\
& & & & & & & \\
& & & & & & & \\
Cumulant & 0.244 & 0.214 & 1.351 & 2.047 & 1.332 & 2.033 & 1.159 \\
Cluster & 0.245 & 0.212 & 1.378 & 2.130 & 1.337 & 2.136 & 1.161 \\
Series expansion & & 0.2135 & 1.587 & 2.485 & 1.587 & 2.485 & 1.587 \\
\hline \hline
\end{tabular}

The relative accuracy of the results obtained also for these Potts systems makes us confident that it should generally make sense to require from approximate transformations to satisfy exact group structure properties, at least as far as the weights have the form (2.4).

We close this section by noting that nothing can be done, in all the above examples, in order to get magnetic exponents $h_{h}^{\left(l, l^{\prime}\right)}$, analogous to the thermal $y_{T}^{\left(l, l^{\prime}\right)}$. The reason lies in the particular symmetry with respect to the $h$ variable, which forces us to assume $q$ and $r$ to be even functions of $h$, thus with a strictly zero derivative at the fixed point.

\section{AN APPLICATION TO NONLINEAR TRANSFORMATIONS}

In this section, we report on some applications of the above ideas to nonlinear transformations of a form generally used in the free-energy optimalization approach. ${ }^{5-7}$ The resuits, in our opinion, are interesting both because they provide explicit examples of situations in which no strict matching can be ob- tained, and because, on the other hand, they can be interpreted in a way which leads to analogous conclusions as in the variational context.

We consider two-dimensional Ising systems and weight functions of the form

$$
\begin{aligned}
P_{q}(\{\vec{\mu}\},\{\vec{\sigma}\})=\prod_{\alpha} \exp \left(q \sum_{i \in \alpha}\left(\vec{\mu}_{\alpha}, \vec{\sigma}_{i}\right)\right. \\
\left.\quad-\ln \operatorname{Tr}_{\vec{\mu}_{\alpha}} \exp q \sum_{i \in \alpha}\left(\vec{\mu}_{\alpha}, \vec{\sigma}_{i}\right)\right),
\end{aligned}
$$

where $\alpha$ runs over three-spin $(l=\sqrt{3})$ and four-spin $(l=2)$ cells in the triangular and square lattices, respectively. The square of $P_{q},\left(P_{q}\right)^{2}$, is then computed according to Eq. (3.1).

The approximations used to compute the transformations associated with $P_{q}$ and $\left(P_{q}\right)^{2}$ are first-order cumulant expansions, with the intracell Hamiltonian of the corresponding spin cells being chosen as unperturbed part.

The plots of $q^{(l)}\left(K^{*}\right)$ and $q^{\left(l^{2}\right)}\left(K^{*}\right)$ for both lat-

\begin{tabular}{|c|c|c|c|c|c|c|c|c|c|}
\hline & Lattice & $l$ & $l^{\prime}$ & $q^{(l)}\left(K_{c}^{*}\right)$ & $K_{c}^{*}$ & $y_{T}^{(l)}$ & $y_{T}^{\left(l^{\prime}\right)}$ & $y_{T}^{\left(l, l^{\prime}\right)}$ & $\begin{array}{c}\text { Exact }^{\mathrm{a}} \text { or series } \\
\text { results }\end{array}$ \\
\hline \multirow[t]{2}{*}{$s=3$} & Triangular & $\sqrt{3}$ & 3 & 0.455 & 0.412 & 1.152 & 1.108 & 0.965 & $K_{c}^{*}=0.421 ; y_{T}=1.174$ \\
\hline & Square & 2 & 3 & 0.343 & 0.648 & 1.295 & 1.252 & 1.064 & $K_{c}^{*}=0.670 ; y_{T}=1.174$ \\
\hline$s=4$ & Square & 2 & 3 & 0.356 & 0.817 & 1.355 & 1.313 & 1.139 & $K_{c}^{*}=0.824 ; y_{T}=1.290$ \\
\hline$s=5$ & & 2 & 3 & 0.367 & 0.947 & 1.396 & 1.356 & 1.193 & $K_{c}^{*}=0.939 ;$ first order ${ }^{c}$ \\
\hline
\end{tabular}

TABLE IV. Results of the matchings for the $s=3,4$, and 5 two-dimensional Potts systems (cumulant approximation). 


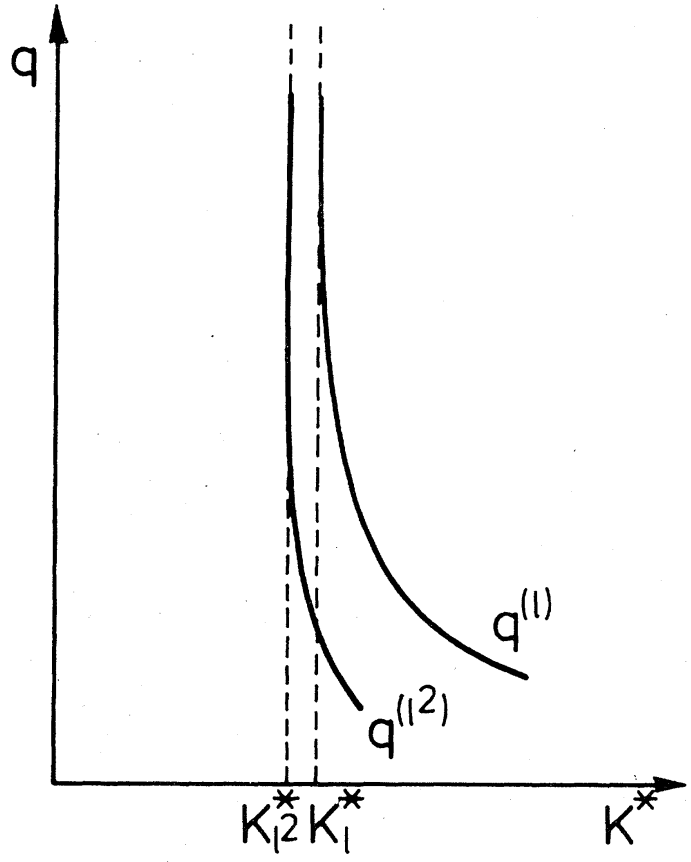

FIG. 2. Schematic behavior of $q^{(l)}$ and $q^{\left(l^{2}\right)}$ in the nonlinear cases. $K_{l}^{*}$ and $K_{l 2}^{*}$ denote the asymptotic values of $K^{*}$ for $q^{(l)}$ and $q^{\left(l^{2}\right)}$ going to infinity.

tices have a shape of the kind schematically represented in Fig. 2, i.e., there is no matching abscissa, unlike that in the previous cases. The behavior of both $q^{(l)}$ and $q^{\left(l^{2}\right)}$ is such that they approach vertical asymptotes at $K_{l}^{*}$ and $K_{l^{2}}^{*}$, respectively, and the absolute value of the difference between the $K^{*}$ 's, which give rise to an identical value of $q^{(l)}$ and $q^{\left(l^{2}\right)}$, is appreciably decreasing with the increasing of this value for $q$, reaching its minimum, $\left|K_{l}^{*}-K_{l^{2}}^{*}\right|$, asymptotically at $q^{(l)}=q^{\left(l^{2}\right)}=\infty$.

This would suggest us to consider as optimal both transformations, when the parameter value is taken to be infinite. Indeed, in both cases, all the transfor- mations, as reported in Table $\mathrm{V}$, show the best agreement of $K^{*}, y_{T}$, and $y_{h}$ with the exact values in this asymptotic situation. At infinite value of $q$, the transformations induced by the weights of the form (5.1) are called majority rule transformations. ${ }^{1}$

It is worth mentioning here that the transformations induced by our $P_{q}$ 's and by their squares, if analyzed from the point of view of free-energy optimalization, in the cumulant approximations considered, also turn out to be optimal for $q=\infty$. This had already been remarked by van Saarloos et al. ${ }^{6}$ and by Barber ${ }^{14}$ for weights like Eq. (5.1), and it can be verified along similar lines in the case of our $\left(P_{q}\right)^{2}$ 's.

\section{SPIN- $\frac{1}{2} X Y$ SYSTEM IN TWO DIMENSIONS}

Unlike the previous ones, the application we want to discuss in this section is within a context in which very little is reliably known from various approximate methods. Our aim here is thus mainly to outline some possibilities and focus some problems, rather than to draw any, even tentative, conclusions.

We are concerned with the spin- $\frac{1}{2} X Y$ model on a triangular lattice, with Hamiltonian

$$
H(\{\vec{\sigma}\})=K \sum_{\langle i j\rangle}\left(\sigma_{i}^{x} \sigma_{j}^{x}+\sigma_{i}^{y} \sigma_{j}^{y}\right),
$$

where the $\sigma$ 's here are Pauli spin- $\frac{1}{2}$ operator components.

One of the few facts about which little doubt seems to exist at present is that the spin- $\frac{1}{2} X Y$ model in two dimensions undergoes some kind of finite temperature phase transition. The particular, continuous symmetry of Eq. (6.1) and the dimensionality $d=2$, however, make it very difficult to predict the nature of this transition. Nevertheless, a recent application of quantum renormalization methods ${ }^{15}$ seems to favor the possibility that the transition is of the same type as that expected, on the basis of other theoretical approaches, for the classical, spin- $\infty, X Y$ system. ${ }^{16,17}$ According to these theories, what one

TABLE V. Critical properties given by the nonlinear transformations at $q=\infty . l=\sqrt{3}$ and 3 , in the triangular and square lattice case, respectively.

\begin{tabular}{|c|c|c|c|c|c|c|c|}
\hline Lattice & $K_{l}^{*}$ & $K_{l^{2}}^{*}$ & Exact $K_{c}^{*}$ & $y_{T}^{(l)}$ & $y_{h}^{(l)}$ & $y_{T}^{\left(I^{2}\right)}$ & $y_{h}{ }^{\left(l^{2}\right)}$ \\
\hline Triangular & 0.335 & 0.316 & 0.275 & 0.882 & 2.034 & 0.899 & 1.857 \\
\hline Square & 0.519 & 0.476 & 0.441 & 1.005 & 2.145 & 1.000 & 2.000 \\
\hline Exact & & & & 1.000 & 1.875 & 1.000 & 1.875 \\
\hline
\end{tabular}


should expect working with a linear renormalization transformation ${ }^{15}$ is to find fixed points with marginal temperature behavior, i.e., with $y_{T}=0$.

This marginality, which implies exponential rather than power law temperature singularities, is connected with the fact that the system, instead of displaying only one isolated critical point, is expected to have a whole line of critical points in the low-temperature region. When applying the quantum real-space renormalization approach to our system, this special behavior of the transformation, i.e., the fixed point with temperature marginality, is never seen with first-order cumulant expansions, but is seen in second order when the transformations themselves acquire a much richer structure. The first-order approximations always predict, for every value of the parameter, an isolated critical point, with a power law temperature singularity.

Thus, in order to test the consistency of these first-order approximations, and to fix the parameter value in the transformations, we have applied our matching procedure.

We have evaluated in a suitable first-order cumulant approximation a linear transformation in which the basic cell is the familiar triangular three-spin cell $(l=\sqrt{3})$; for the details of this kind of calculations and, in general, for the formulation of renormalization approaches in the quantum context, we refer to Ref. 15 and to other references quoted therein. By a considerable computational effort, in this case, we have then been able to compute the square of this transformation, with nine-spin cells $(l=3)$, in an analogous kind of approximation. This essentially amounted to diagonalizing numerically the $2^{9} \times 2^{9}$ matrix representing the unperturbed intracell Hamiltonian of a nine-spin cell; exploiting the symmetry of Eq. (6.1) and of the cell, the maximum size of the blocks to be diagonalized reduced to $42 \times 42$.

Unlike in all the numerous cases discussed in Sec. IV, for this model the matching of the fixed points

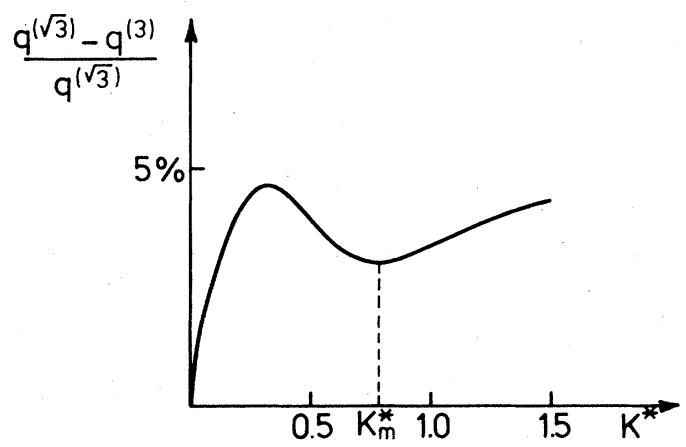

FIG. 3. Relative difference $\left(q^{(\sqrt{3})}-q^{(3)}\right) / q^{(\sqrt{3})}$ between $q^{(\sqrt{3})}$ and $q^{(3)}$ for the spin- $\frac{1}{2} X Y$ system as a function of $K^{*}$. $K_{m}^{*}$ denotes the abscissa of the relative minimum. turned out not to have taken place, i.e., $q=q^{(l)}\left(K^{*}\right)$ and $q=q^{\left(l^{2}\right)}\left(K^{*}\right)$ have no intersection for finite $K^{*}$. The situation is graphically reported in Fig. 3, where the percentual deviation $\left(q^{(l)}-q^{\left(l^{2}\right)}\right)\left(q^{(l)}\right)^{-1}$ is given as a function of $K^{*}$, in a reasonably large interval. Instead of crossing the $K^{*}$ axis at a finite abscissa $K_{c}^{*}$, this quantity has a positive $(\approx 3 \%)$ minimum at $K^{*}=K_{m}^{*} \cong 0.8$.

We thus see that the transformations considered in this approximation never strictly satisfy, at their fixed points, the exact group structure relationship which follows from their definition. The situation is similar, in some respects, to that of the previous section, with the difference, however, that there the curves $q=q^{(l)}\left(K^{*}\right)$ and $q=q^{\left(l^{2}\right)}\left(K^{*}\right)$ had veritcal asymptotes, and that the situation closest to matching was reached there for a boundary value of $q(q=\infty)$.

A possible tentative conclusion is that the application of the method indicates a location for the critical point around the value $K_{m}^{*}$. In fact, when working with higher-order approximations, a fixed point, but now with marginal temperature eigenvalue, can always be located around $0.6 \sim 0.7$.

Just as a conjecture, we would also like to interpret the above result as telling us that, as far as the critical behavior is concerned, the predictions obtained by the above approximate transformations, even at $K^{*} \approx K_{m}^{*}$, are not very reliable.

In any case, the very peculiar behavior found in this case further enforces our suspicion that the transition undergone by the spin- $\frac{1}{2} X Y$ model is of a somewhat unusual nature, quite different from those of Potts-like systems.

\section{CONCLUDING REMARKS}

In the literature on the real-space renormalization approach to critical phenomena, up to now little attention has been paid to possible consequences of the group structure properties of the transformations.

The present paper was to some extent intended to fill this gap. We have shown that these group structure properties, if properly used, can play an important practical and conceptual role in the whole approach; combined with very simple approximations they did yield rather accurate predictions of the critical parameters in most of the cases. Indeed, one of the advantages of our method is that it is not necessary to use sophisticated approximations for obtaining this accuracy.

It is, however, natural to ask how the described schemes could eventually be adapted to more complicated transformations like, e.g., those that are generated in higher dimensional even interaction spaces by second-order cumulant or many-cell cluster ap- 
proximations. $^{1}$

For a satisfactory treatment of these cases, one should in general work with weight factors containing as many independent parameters as there are interaction couplings, in such a way as to obtain eventually the complete, multidimensional matching of the fixed points. This would of course make the whole procedure quite complicated and of no practical use.

We have tried to proceed in a simplified way, by looking for a matching of the critical nearest-neighbor couplings only; we did this with the previously defined one-parameter transformations $f^{(\sqrt{3})}$ and $f^{(3)}$, in the triangular lattice, computed now up to second order in the cumulant expansions. Contrary to what might be expected, however, the desired matching did not take place in this particular case, probably be- cause of the linear extrapolations involved in the actual computation of the critical couplings. ${ }^{1}$

In order to make the procedure compatible with complicated approximations, a more practical, and perhaps more successful direction to follow could be to use still one-parameter dependent weights and to convert the transformations from the start into effective ones, working on the nearest-neighbor coupling only. A reduction of this type, taking into account further neighbor interactions in some mean-field approximation and disregarding the effect of multispin couplings, has been recently proposed as a tool for getting tractable transformations. ${ }^{18}$

Efforts in this direction would hopefully provide us with further insight into the open problems concerning the two-dimensional spin- $\frac{1}{2} X Y$ system.
"On leave of absence from Istituto di Fisica e Unità G.N.S.M. del C. N. R., Università di Padova, Padova, Italy.

'Th. Niemeijer and J. M. J. van Leeuwen, in Phase Transitions and Critical Phenomena, edited by C. Domb and M. S. Green (Academic, London, 1976), Vol. 6, p. 425.

${ }^{2}$ For a review of more recent developments, see also J. M. J. van Leeuwen, Ann. Israel Phys. Soc. 2, (1), 174 (1978).

${ }^{3}$ K. G. Wilson, Rev. Mod. Phys. 47, 773 (1975); and T. L. Bell and K. G. Wilson, Phys. Rev. B 11, 3431 (1975).

${ }^{4}$ A. Fiorenzato, A. L. Stella, and F. Toigo, Phys. Lett. A 69 , 127, 471(E) (1978).

${ }^{5}$ L. P. Kadanoff, A. Houghton, and M. C. Yalabik, J. Stat. Phys. 14, 171 (1976).

${ }^{6}$ W. van Saarloos, J. M. J. van Leeuwen, and A. M. M. Pruisken, Physica (Utrecht) A 92, 323 (1978).

${ }^{7}$ S. J. Shenker, L. P. Kadanoff, and A. M. M. Pruisken (unpublished).

${ }^{8}$ In this paper we obviously assume the practical attitude of giving for granted the existence and regularity of mappings like that in Eq. (2.3); for a rigorous discussion of this point we refer the reader to R. B. Griffiths and P. A. Pearce, Phys. Rev. Lett. 41, 917 (1978).

${ }^{9} \mathrm{The}$ symbolic value of the notations in Eq. (3.4) should be noted: we know that the "exact" transformations act on infinite-dimensional interaction spaces.

${ }^{10} \mathrm{O}$ course, nothing a priori guarantees the existence of such an intersection. In Secs. V and VI we meet some situations in which it does not exist; nevertheless, also in these cases some conclusions can be drawn.

${ }^{11}$ R. J. Baxter, H. N. V. Temperley, and S. E. Ashley, Proc. R. Soc. London A 358, 535 (1978).

${ }^{12}$ R. Zwanzig and J. Ramshaw, J. Phys. A 10, 65 (1977).

${ }^{13}$ R. J. Baxter, J. Phys. C $\underline{6}$, L445 (1973).

${ }^{14}$ M. N. Barber, J. Phys. A 10,1187 (1977).

${ }^{15}$ R. Dekeyser, M. Reynaert, A. L. Stella, and F. Toigo, Phys. Rev. B 18, 3486 (1978).

16J. M. Kosterlitz and D. J.Thouless, J. Phys. C $\underline{6}, 1181$ (1973).

17J. José, L. P. Kadanoff, S. Kirkpatrick, and D. R. Nelson, Phys. Rev. B 16, 1217 (1977).

${ }^{18}$ A. Casher and M. Schwartz, Phys. Rev. B 18,3440 (1978) 